\title{
Endophytic Fungi of Olive Tree
}

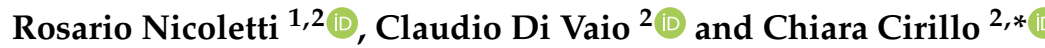 \\ 1 Council for Agricultural Research and Economics, Research Centre for Olive, Fruit and Citrus Crops, \\ 81100 Caserta, Italy; rosario.nicoletti@crea.gov.it \\ 2 Department of Agricultural Sciences, University of Naples Federico II, 80055 Portici, Italy; divaio@unina.it \\ * Correspondence: chiara.cirillo@unina.it
}

Received: 4 August 2020; Accepted: 28 August 2020; Published: 30 August 2020

\begin{abstract}
In addition to the general interest connected with investigations on biodiversity in natural contexts, more recently the scientific community has started considering occurrence of endophytic fungi in crops in the awareness of the fundamental role played by these microorganisms on plant growth and protection. Crops such as olive tree, whose management is more and more frequently based on the paradigm of sustainable agriculture, are particularly interested in the perspective of a possible applicative employment, considering that the multi-year crop cycle implies a likely higher impact of these symbiotic interactions. Aspects concerning occurrence and effects of endophytic fungi associated with olive tree (Olea europaea) are revised in the present paper.
\end{abstract}

Keywords: Olea europaea; endophytes; antagonism; defensive mutualism; plant growth promotion; bioactive compounds

\section{Introduction}

After evidence resulting from the manifold investigations carried out in the last decades, the awareness that endophytic fungi are constantly associated with plants and remarkably influence their ecological fitness has significantly increased. In fact, the original boost concerning natural ecosystems incited by the general theoretical intent to exploit all components of biodiversity, basically as a source of novel bioactive products, has more recently extended to crops. Within agricultural contexts, the role of the endophytic microbiota, or endosphere, is more consistent in orchards, where the time factor confers higher impact to the establishment of an equilibrium among the species which are part of the tree biocoenosis, as well as to its eventual disruption [1].

The extent at which the accumulating knowledge on the beneficial effects of endophytic microorganisms may have a practical impact in tree crop management, and further progresses can be achieved, is largely dependent on the opportunity by the scientific community and actors in the field to access it in an organized form. In this perspective, the state of the art of research concerning occurrence and effects of endophytic fungi associated with olive tree (Olea europaea) are revised in the present paper.

\section{Relevance of Microorganisms for a Sustainable Management in Olive Growing}

The Mediterranean Basin landscape and culture have been shaped by olive tree since ancient times, but the ecological importance of this tree has only recently been acknowledged [2,3]. In the semiarid Mediterranean agricultural lands, new approaches in fruit orchard management have been forced by environmental constrains, such as soil degradation and water shortage, and agronomical techniques that may be able to improve or preserve soil quality and fertility, other than plant health, have gained particular importance [4-7]. Modern intensification in olive cultivation practices is causing increased incidence and severity of olive pests and diseases; whereas sustainable management systems can 
positively affect soil biochemical characteristics and soil microbial biodiversity [8,9], and contribute to improve landscape stability, mainly in the rising condition of abandoned olive groves [3]. Thus, year by year a fast-growing percentage of the growers' incomes is invested in agrochemicals, to promote olive tree growth, to control plant pathogens, and to increase the olive yield and quality, simultaneously generating a great public concern on the negative effects of the agrochemicals use on the environment, on the ecosystem's biodiversity, and human and animal health [10]. Consequently, several efforts have been done on the development of eco-friendly cultivation practices suitable to sustainable disease control by ameliorating olive tree health and productivity through methods and strategies that promote soil biological processes, decrease agricultural inputs, and improve soil structure and fertility [9].

The diversity of microorganisms associated with plants may stimulate their growth and induce tolerance mechanisms helping plants to counteract adverse environmental conditions. In arid and semiarid environments, crops are facing environmental constraints due to climate-change-driven rising temperatures, changes in rainfall frequency, and occurrence of extreme events [11]. These habitat-elicited stresses may reduce crop productivity and lead to soil erosion and degradation. Plants dwelling in such environments have developed mechanisms helping them to mitigate and counteract abiotic stress. Microorganisms of the rhizosphere can play a pivotal role in health and growth of olive tree too, by establishing strong relationships with the root system that enable plants to grow in limiting conditions, such as water scarcity, salinity, low soil fertility, and so on. In addition to studies on the intrinsic ability of olive tree to adapt to adverse environmental conditions [12], a significant research activity has been performed on rhizosphere microbes providing increased tolerance to host plants under abiotic stress, mainly focusing on plant growth promoting rhizobacteria and arbuscular mycorrhizal fungi $[13,14]$. Moreover, fungi and actinomycetes have been recognized as able to use root exudates as a carbon source, supplying plants with promptly assimilable nitrates, and playing a crucial role in the maintenance of soil health, besides exerting antagonistic effects on root pathogens [5].

In the plant holobiont system, these beneficial effects are integrated by the microbial component of the endosphere. Endophyte colonization of plants has been recognized to involve a sequence of cross-talking signals that allow the onset of compatible interactions. Once the interrelation has established, endophytes increase stress tolerance through the stress-responsive gene induction/expression, reactive oxygen species and anti-stress metabolite synthesis [15]. Under abiotic stress conditions, endophytic fungi have been reported to produce plant hormones and compatible solutes that maintain integrity and promote growth of the host. Moreover, they are known to protect their host plants against biotic adversities through the production of bioactive compounds and the stimulation of the defense reaction [16]. As soon as interactions between endophytes and plants have been disclosed, it has been argued that they can be exploited for the development of innovative applications in sustainable but still highly productive cultivation systems ([17], and literature therein), similarly to the better known other groups of microbes. As a result of the rising demand for organic agricultural products, perspectives for the application of these microorganisms as potential biopesticides and biofertilizers have become more consistent in the olive sector too [18], along with an incremental interest for the search and identification of species-specific endophytes [19].

\section{Occurrence and Ecological Implications of Endophytic Fungi of Olive Tree}

Tables 1 and 2 list records concerning occurrence of endophytic fungi in olive tree as inferred from examination of the available literature and GenBank accessions. The first table, dedicated to Ascomycota, is much more numerous; in fact, it includes 245 entries, 116 of which (approx. $47 \%$ ) are identified at the species level. Such a low proportion can be explained considering that rDNA-ITS sequences are not able to resolve species ascription within many fungal genera [20]. On the other hand, it could reflect the possible existence of novel species, which is quite a common outcome of investigations on endophytic fungi. The same inference could apply to the Basidiomycota series of records, where identification at the species level is occasional, and was achieved for just 7 out of 
37 entries (about 19\%). Finally, taxa belonging to the Mucoromycota appear to be quite infrequent (Table 2).

Table 1. Endophytic Ascomycota reported from Olea europaea.

\begin{tabular}{|c|c|c|c|}
\hline Endophyte $^{1}$ & Plant Part & Country & Reference \\
\hline \multirow{2}{*}{$\begin{array}{c}\text { Absconditella sp. } \\
\text { Acaulium sp. }\end{array}$} & branch & Salento, Italy & [21] \\
\hline & root & Córdoba, Spain & [22] \\
\hline \multirow{4}{*}{ Acremonium sp. } & leaf, twig & Sicily, Italy & [23] \\
\hline & branch, leaf & Salento, Italy & [21] \\
\hline & leaf, root & Bragança district, Portugal & [24] \\
\hline & leaf & Trás-os-Montes, Portugal & [25] \\
\hline \multirow[t]{3}{*}{ Alternaria alternata } & leaf & Alentejo, Portugal & [26] \\
\hline & twig & Mirandela, Portugal & [27] \\
\hline & fruit & Karaburun, Turkey & (GenBank) \\
\hline Alternaria arborescens & leaf & Bragança district, Portugal & [24] \\
\hline Alternaria brassicae & fruit & Mirandela, Portugal & [28] \\
\hline Alternaria compacta & leaf & Alentejo, Portugal & [26] \\
\hline Altenaria consortialis & root & Bragança district, Portugal & [24] \\
\hline \multirow{2}{*}{ Alternaria infectoria } & leaf & Alentejo, Portugal & [26] \\
\hline & twig & Mirandela, Portugal & [27] \\
\hline Alternaria murispora & leaf & Alentejo, Portugal & [26] \\
\hline Alternaria preussii & leaf & Mirandela, Portugal & [29] \\
\hline \multirow[t]{4}{*}{ Alternaria solani } & twig & Mirandela, Portugal & [27] \\
\hline & stem & Majorca, Spain & [30] \\
\hline & leaf, twig & Sicily, Italy & [23] \\
\hline & leaf & Evora, Portugal & [31] \\
\hline \multirow[t]{4}{*}{ Alternaria sp. } & fruit & Mirandela, Portugal & [28] \\
\hline & leaf, twig & Mirandela, Portugal & {$[27,29]$} \\
\hline & leaf & Alentejo, Portugal & [32] \\
\hline & branch, leaf & Salento, Italy & [21] \\
\hline \multirow{2}{*}{ Alternaria tenuissima } & stem, xylem & Majorca, Spain & [30] \\
\hline & twig & Mirandela, Portugal & [27] \\
\hline Anthostomella leucospermi & leaf, twig & Mirandela, Portugal & {$[27,29]$} \\
\hline Arcopilus aureus & leaf & Alentejo, Portugal & {$[26]$} \\
\hline Arthrinium phaeospermum & stem, xylem & Majorca, Spain & [30] \\
\hline \multirow{2}{*}{ Arthrinium sp. } & leaf & Alentejo, Portugal & [32] \\
\hline & leaf & Salento, Italy & [21] \\
\hline Ascochyta sp. & leaf, twig & Sicily, Italy & [23] \\
\hline \multirow[t]{4}{*}{ Ascochytulina deflectens } & stem, xylem & Majorca, Spain & [30] \\
\hline & leaf & Piracicaba, Brazil & [33] \\
\hline & fruit & Bragança district, Portugal & [34] \\
\hline & leaf, twig & Mirandela, Portugal & {$[27,29]$} \\
\hline \multirow[t]{4}{*}{ Aspergillus sp. } & leaf & Alentejo, Portugal & {$[26,32]$} \\
\hline & root & Córdoba, Spain & [22] \\
\hline & xylem & Apulia, Italy & [35] \\
\hline & branch, leaf & Salento, Italy & [21] \\
\hline Aspergillus stellatus & twig & Sicily, Italy & [23] \\
\hline \multirow[t]{2}{*}{ Aspergillus tubingensis } & root & Siwa oasis, Egypt & [36] \\
\hline & stem, xylem & Majorca, Spain & [30] \\
\hline \multirow[t]{3}{*}{ Aureobasidium pullulans } & leaf, twig & Sicily, Italy & [23] \\
\hline & leaf & Alentejo, Portugal & [26] \\
\hline & leaf, twig & Mirandela, Portugal & [29] \\
\hline \multirow{2}{*}{ Aureobasidium sp. } & leaf & Alentejo, Portugal & [32] \\
\hline & branch, leaf & Salento, Italy & [21] \\
\hline Bartalinia sp. & fruit & Karaburun, Turkey & (GenBank) \\
\hline Berkeleyomyces basicola & root & Bragança district, Portugal & [24] \\
\hline Biatora sp. & branch & Salento, Italy & [21] \\
\hline
\end{tabular}


Table 1. Cont.

\begin{tabular}{|c|c|c|c|}
\hline Endophyte $^{1}$ & Plant Part & Country & Reference \\
\hline \multirow{4}{*}{ Biscogniauxia mediterranea } & flower buds & Bragança district, Portugal & [34] \\
\hline & fruit & Mirandela, Portugal & [28] \\
\hline & leaf & Alentejo, Portugal & [26] \\
\hline & leaf, twig & Mirandela, Portugal & {$[27,29]$} \\
\hline Biscogniauxia nummularia & shoot & Ljubljana, Slovenia & [37] \\
\hline \multirow[t]{2}{*}{ Botryosphaeria sp. } & leaf, twig & Sicily, Italy & [23] \\
\hline & leaf, twig & Sicily, Italy & [23] \\
\hline \multirow[t]{2}{*}{ Botrytis cinerea } & leaf & Alentejo, Portugal & [26] \\
\hline & leaf, twig & Mirandela, Portugal & {$[27,29]$} \\
\hline \multirow{2}{*}{ Botrytis sp. } & leaf & Alentejo, Portugal & {$[32]$} \\
\hline & branch & Salento, Italy & [21] \\
\hline Cadophora luteo-olivacea & root & Bragança district, Portugal & [24] \\
\hline \multirow{2}{*}{ Camarosporium sp. } & leaf, twig & Sicily, Italy & [23] \\
\hline & leaf, twig & Mirandela, Portugal & {$[27,29]$} \\
\hline Canalisporium sp. & root & Córdoba, Spain & [22] \\
\hline \multirow{2}{*}{ Candida sp. } & twig & Sicily, Italy & [23] \\
\hline & branch, leaf & Salento, Italy & [21] \\
\hline Capnobotryella sp. & branch & Salento, Italy & [21] \\
\hline Catenulostroma sp. & branch, leaf & Salento, Italy & [21] \\
\hline Catillaria sp. & branch & Salento, Italy & [21] \\
\hline Ceratocystis sp. & leaf, twig & Sicily, Italy & [23] \\
\hline Cercospora sp. & branch & Salento, Italy & {$[21]$} \\
\hline Ceuthospora sp. & xylem & Apulia, Italy & [35] \\
\hline \multirow[t]{2}{*}{ Chaetomium globosum } & shoot & Ljubljana, Slovenia & {$[37]$} \\
\hline & leaf & Sicily, Italy & [23] \\
\hline \multirow{3}{*}{ Chaetomium sp. } & leaf & Evora, Portugal & [31] \\
\hline & shoot & Ljubljana, Slovenia & [37] \\
\hline & leaf & Alentejo, Portugal & [32] \\
\hline \multirow{2}{*}{ Chalara sp. } & leaf, twig & Sicily, Italy & [23] \\
\hline & leaf & Alentejo, Portugal & {$[32]$} \\
\hline Chalastospora gossypii & leaf, twig & Mirandela, Portugal & {$[27,29]$} \\
\hline Chromelosporium carneum & leaf, twig & Mirandela, Portugal & {$[27,29]$} \\
\hline Ciboria sp. & leaf & Salento, Italy & {$[21]$} \\
\hline Cladophialophora sp. & root & Córdoba, Spain & [22] \\
\hline \multirow{2}{*}{ Cladosporium cladosporioides } & leaf & Alentejo, Portugal & {$[26]$} \\
\hline & fruit & Mirandela, Portugal & [28] \\
\hline Cladosporium cucumerinum & fruit & Mirandela, Portugal & [28] \\
\hline Cladosporium delicatulum & leaf & Alentejo, Portugal & [26] \\
\hline Cladosporium herbarum & leaf & Alentejo, Portugal & [26] \\
\hline $\begin{array}{c}\text { Cladosporium } \\
\text { pseudocladosporioides }\end{array}$ & leaf & Alentejo, Portugal & [26] \\
\hline Cladosporium ramotenellum & fruit & Karaburun, Turkey & (GenBank) \\
\hline \multirow{6}{*}{ Cladosporium sp. } & leaf, twig & Sicily, Italy & {$[23]$} \\
\hline & shoot & Ljubljana, Slovenia & [37] \\
\hline & fruit & Mirandela, Portugal & [28] \\
\hline & leaf, twig & Mirandela, Portugal & {$[27,29]$} \\
\hline & xylem & Apulia, Italy & {$[35]$} \\
\hline & leaf & Salento, Italy & {$[21]$} \\
\hline Cladosporium sphaerospermum & shoot & Ljubljana, Slovenia & [37] \\
\hline Cladosporium tenellum & leaf & Alentejo, Portugal & [26] \\
\hline Cladosporium tenuissimum & stem, xylem & Majorca, Spain & {$[30]$} \\
\hline Clonostachys rosea & root & Bragança district, Portugal & [24] \\
\hline \multirow{2}{*}{ Colletotrichum acutatum } & fruit & Gioia Tauro area, Italy & [38] \\
\hline & twig & Portugal & (GenBank) \\
\hline Colletotrichum nymphaeae & leaf & Alentejo, Portugal & [26] \\
\hline
\end{tabular}


Table 1. Cont.

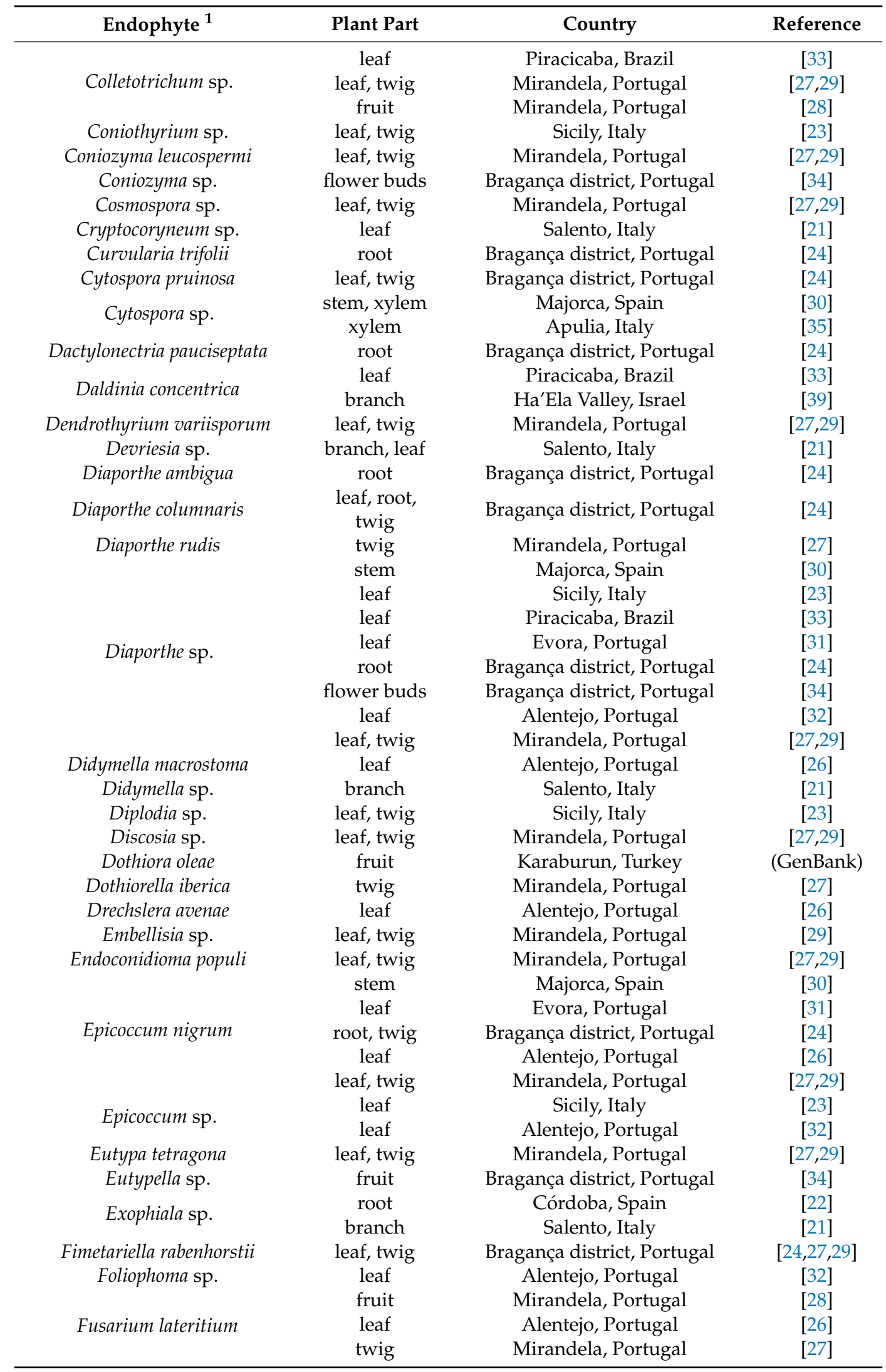


Table 1. Cont.

\begin{tabular}{|c|c|c|c|}
\hline Endophyte ${ }^{1}$ & Plant Part & Country & Reference \\
\hline Fusarium musae & leaf & Alentejo, Portugal & [26] \\
\hline \multirow{3}{*}{ Fusarium oxysporum } & root & Bragança district, Portugal & {$[24,40]$} \\
\hline & twig & Mirandela, Portugal & {$[27]$} \\
\hline & leaf & Evora, Portugal & {$[31]$} \\
\hline \multirow{3}{*}{ Fusarium sp. } & leaf, twig & Mirandela, Portugal & {$[27,29]$} \\
\hline & leaf & Alentejo, Portugal & [32] \\
\hline & xylem & Apulia, Italy & {$[35]$} \\
\hline Fusarium tricinctum & leaf & Alentejo, Portugal & [26] \\
\hline Fusarium verticillioides & leaf & Alentejo, Portugal & [26] \\
\hline Geopyxis sp. & leaf, twig & Mirandela, Portugal & [29] \\
\hline Gibberella avenacea & fruit & Mirandela, Portugal & [28] \\
\hline \multirow{2}{*}{ Gibberella sp. } & twig & Mirandela, Portugal & [27] \\
\hline & branch & Salento, Italy & [21] \\
\hline Gloeosporium sp. & leaf & Sicily, Italy & [23] \\
\hline Gloeotinia granigena & leaf & Alentejo, Portugal & [26] \\
\hline Heydenia alpina & twig & Mirandela, Portugal & [27] \\
\hline Heydenia sp. & leaf, twig & Mirandela, Portugal & {$[27,29]$} \\
\hline Homortomyces sp. & leaf, twig & Mirandela, Portugal & [29] \\
\hline \multirow{2}{*}{ Hormonema sp. } & stem & Majorca, Spain & [30] \\
\hline & leaf & Salento, Italy & [21] \\
\hline Hortaea sp. & branch, leaf & Salento, Italy & {$[21]$} \\
\hline Hyalodendriella betulae & leaf, twig & Mirandela, Portugal & {$[27,29]$} \\
\hline Hyperphyscia sp. & branch & Salento, Italy & {$[21]$} \\
\hline Hypoxylon sp. & stem & Majorca, Spain & [30] \\
\hline Ilyonectria sp. & leaf, twig & Mirandela, Portugal & [29] \\
\hline Kabatina sp. & xylem & Majorca, Spain & {$[30]$} \\
\hline Lecania sp. & branch, leaf & Salento, Italy & [21] \\
\hline Lecanora sp. & branch, leaf & Salento, Italy & {$[21]$} \\
\hline Lecidella sp. & branch, leaf & Salento, Italy & [21] \\
\hline Lecythophora sp. & leaf, twig & Mirandela, Portugal & [29] \\
\hline Leimonis sp. & branch & Salento, Italy & [21] \\
\hline Leptosphaerulina americana & leaf & Alentejo, Portugal & [26] \\
\hline Leptosphaerulina australis & leaf & Alentejo, Portugal & [26] \\
\hline Leptosphaerulina saccharicola & leaf & Alentejo, Portugal & [26] \\
\hline Leptosphaerulina trifolii & leaf & Alentejo, Portugal & [26] \\
\hline Libertasomyces platani & xylem & Apulia, Italy & [35] \\
\hline Lophiostoma corticola & root & Bragança district, Portugal & [24] \\
\hline Lophiostoma sp. & branch, leaf & Salento, Italy & [21] \\
\hline Macrophomina phaseolina & root & Bragança district, Portugal & [24] \\
\hline Macrophomina sp. & root & Córdoba, Spain & [22] \\
\hline Microsphaeropsis arundinis & root & Bragança district, Portugal & [24] \\
\hline Microsphaeropsis proteae & leaf & Mirandela, Portugal & {$[27,29]$} \\
\hline \multirow{2}{*}{ Microsphaeropsis sp. } & stem & Majorca, Spain & {$[30]$} \\
\hline & leaf, twig & Mirandela, Portugal & {$[27,29]$} \\
\hline Minimelanolocus sp. & root & Córdoba, Spain & [22] \\
\hline Mycocalicium victoriae & xylem & Apulia, Italy & [35] \\
\hline Mycosphaerella sp. & leaf, twig & Mirandela, Portugal & [29] \\
\hline Naevala sp. & leaf & Salento, Italy & [21] \\
\hline Nemania aenea & leaf & Mirandela, Portugal & [29] \\
\hline Nemania sp. & leaf, twig & Mirandela, Portugal & [29] \\
\hline Neocamarosporium sp. & leaf, twig & Mirandela, Portugal & [29] \\
\hline Neocatenulostroma sp. & branch, leaf & Salento, Italy & {$[21]$} \\
\hline
\end{tabular}


Table 1. Cont.

\begin{tabular}{|c|c|c|c|}
\hline Endophyte ${ }^{1}$ & Plant Part & Country & Reference \\
\hline \multirow{2}{*}{ Neocosmospora solani } & root & Siwa oasis, Egypt & [36] \\
\hline & twig & Mirandela, Portugal & [27] \\
\hline \multirow{3}{*}{$\begin{array}{l}\text { Neodevriesia sp. } \\
\text { Neofabraea kienholzii }\end{array}$} & branch, leaf & Salento, Italy & [21] \\
\hline & leaf, twig & Mirandela, Portugal & {$[27,29]$} \\
\hline & twig & Sicily, Italy & {$[23]$} \\
\hline \multirow{4}{*}{ Neofabraea sp. } & fruit & Mirandela, Portugal & [28] \\
\hline & leaf, twig & Mirandela, Portugal & {$[27,29]$} \\
\hline & leaf & Alentejo, Portugal & {$[26]$} \\
\hline & leaf & Alentejo, Portugal & [26] \\
\hline \multirow[t]{2}{*}{ Neofabraea vagabunda } & fruit & Mirandela, Portugal & {$[28,34]$} \\
\hline & leaf, twig & Mirandela, Portugal & [27] \\
\hline Neofusicoccum sp. & leaf & Alentejo, Portugal & [32] \\
\hline \multirow{3}{*}{$\begin{array}{l}\text { Neophaeomoniella sp. } \\
\text { Neosartorya sp. } \\
\text { Neosetophoma } \mathrm{sp} .\end{array}$} & branch, leaf & Salento, Italy & [21] \\
\hline & leaf & Alentejo, Portugal & [26] \\
\hline & leaf & Salento, Italy & [21] \\
\hline \multirow{2}{*}{ Nigrospora oryzae } & stem & Majorca, Spain & [30] \\
\hline & leaf & Evora, Portugal & [31] \\
\hline \multirow{2}{*}{ Nigrospora sp. } & leaf & Piracicaba, Brazil & [33] \\
\hline & leaf & Alentejo, Portugal & [32] \\
\hline \multirow{3}{*}{$\begin{array}{l}\text { Ochrocladosporium sp. } \\
\text { Paecilomyces variotii } \\
\text { Paecilomyces verrucosus }\end{array}$} & leaf, twig & Mirandela, Portugal & [29] \\
\hline & xylem & Bisignano, Italy & [41] \\
\hline & root & Bragança district, Portugal & [24] \\
\hline \multirow{2}{*}{ Paraconiothyrium sp. } & leaf, twig & Mirandela, Portugal & [29] \\
\hline & xylem & Apulia, Italy & [35] \\
\hline Paraphaeosphaeria sporulosa & root & Bragança district, Portugal & [24] \\
\hline \multirow{2}{*}{$\begin{array}{c}\text { Paraphoma chrysanthemicola } \\
\text { Paraphoma sp. }\end{array}$} & leaf, root & Bragança district, Portugal & [24] \\
\hline & root & Bragança district, Portugal & [24] \\
\hline Parastagonospora avenae & twig & Mirandela, Portugal & [27] \\
\hline \multirow{2}{*}{ Penicillium canescens } & root, twig & Bragança district, Portugal & [24] \\
\hline & leaf & Trás-os-Montes, Portugal & {$[25]$} \\
\hline \multirow{2}{*}{ Penicillium chrysogenum } & leaf & Siwa oasis, Egypt & [42] \\
\hline & fruit & Karaburun, Turkey & (GenBank) \\
\hline Penicillium citrinum & fruit & Siwa oasis, Egypt & [36] \\
\hline \multirow{2}{*}{ Penicillium commune } & leaf, twig & Bragança district, Portugal & {$[24,43]$} \\
\hline & leaf & Trás-os-Montes, Portugal & [25] \\
\hline Penicillium echinulatum & leaf & Alentejo, Portugal & [26] \\
\hline Penicillium expansum & leaf & Alentejo, Portugal & [26] \\
\hline Penicillium glabrum & twig & Mirandela, Portugal & [27] \\
\hline Penicillium restrictum & leaf, root & Bragança district, Portugal & [24] \\
\hline \multirow[t]{3}{*}{ Penicillium roseopurpureum } & root & Bragança district, Portugal & {$[24,40]$} \\
\hline & stem, xylem & Majorca, Spain & {$[30]$} \\
\hline & leaf, twig & Sicily, Italy & [23] \\
\hline \multirow[t]{3}{*}{ Penicillum sp. } & leaf, twig & Mirandela, Portugal & {$[27,29]$} \\
\hline & xylem & Apulia, Italy & {$[35]$} \\
\hline & branch, leaf & Salento, Italy & [21] \\
\hline Penicillium spinulosum & leaf & Alentejo, Portugal & [26] \\
\hline \multirow{2}{*}{$\begin{array}{l}\text { Pestalotiopsis guepinii } \\
\text { Pestalotiopsis sp. }\end{array}$} & stem & Majorca, Spain & [30] \\
\hline & leaf, twig & Mirandela, Portugal & {$[27,29]$} \\
\hline Phaeoacremonium sp. & root & Córdoba, Spain & [22] \\
\hline \multirow{2}{*}{$\begin{array}{l}\text { Phaeococcomyces sp. } \\
\text { Phaeohelotium sp. }\end{array}$} & branch, leaf & Salento, Italy & [21] \\
\hline & branch & Salento, Italy & [21] \\
\hline \multirow{2}{*}{ Phaeomoniella sp. } & leaf, twig & Mirandela, Portugal & {$[27,29]$} \\
\hline & branch, leaf & Salento, Italy & {$[21]$} \\
\hline Phaeosphaeria sp. & leaf, twig & Mirandela, Portugal & {$[27,29]$} \\
\hline Phaeothecoidea sp. & branch, leaf & Salento, Italy & [21] \\
\hline Phoma herbarum & leaf & Alentejo, Portugal & [26] \\
\hline
\end{tabular}


Table 1. Cont.

\begin{tabular}{|c|c|c|c|}
\hline Endophyte $^{1}$ & Plant Part & Country & Reference \\
\hline \multirow{3}{*}{ Phoma sp. } & stem, xylem & Majorca, Spain & [30] \\
\hline & leaf, twig & Sicily, Italy & [23] \\
\hline & leaf, twig & Mirandela, Portugal & {$[27,29]$} \\
\hline Phyllosticta sp. & leaf & Piracicaba, Brazil & [33] \\
\hline Pichia sp. & branch & Salento, Italy & [21] \\
\hline Pithomyces chartarum & xylem & Apulia, Italy & [35] \\
\hline Plectania rhytidia & twig & Mirandela, Portugal & [27] \\
\hline Plectania sp. & leaf, twig & Mirandela, Portugal & [29] \\
\hline Pleospora herbarum & stem & Majorca, Spain & [30] \\
\hline Pleospora sp. & leaf, twig & Sicily, Italy & [23] \\
\hline Pleurophoma sp. & stem & Majorca, Spain & [30] \\
\hline Podospora sp. & root & Bragança district, Portugal & [24] \\
\hline \multirow[t]{2}{*}{ Preussia africana } & leaf & Alentejo, Portugal & [26] \\
\hline & leaf & Sicily, Italy & [23] \\
\hline \multirow{3}{*}{ Preussia sp. } & shoot & Ljubljana, Slovenia & [37] \\
\hline & leaf, twig & Mirandela, Portugal & [29] \\
\hline & branch, leaf & Salento, Italy & [21] \\
\hline Prosthemium sp. & leaf, twig & Mirandela, Portugal & {$[27,29]$} \\
\hline Pseudocamarosporium sp. & xylem & Apulia, Italy & {$[35]$} \\
\hline \multirow{2}{*}{ Pseudocercospora sp. } & leaf, twig & Mirandela, Portugal & {$[27,29]$} \\
\hline & branch, leaf & Salento, Italy & [21] \\
\hline Pseudocosmospora vilior & root & Bragança district, Portugal & [24] \\
\hline Pseudophaeomoniella oleae & xylem & Apulia, Italy & [35] \\
\hline \multirow{2}{*}{ Pseudophaeomoniella sp. } & leaf, twig & Mirandela, Portugal & [29] \\
\hline & xylem & Apulia, Italy & [35] \\
\hline Purpureocillium lilacinum & root & Bragança district, Portugal & {$[24,40]$} \\
\hline Purpureocillium sp. & root & Córdoba, Spain & [22] \\
\hline Pycnidiophora sp. & leaf, twig & Mirandela, Portugal & [29] \\
\hline Pyrenochaeta sp. & leaf, twig & Mirandela, Portugal & {$[27,29]$} \\
\hline Pyronema domesticum & leaf, twig & Mirandela, Portugal & {$[27,29]$} \\
\hline Pyrrhospora sp. & branch, leaf & Salento, Italy & {$[21]$} \\
\hline Rachicladosporium sp. & branch & Salento, Italy & [21] \\
\hline \multirow{2}{*}{ Ramularia sp. } & stem & Majorca, Spain & [30] \\
\hline & branch, leaf & Salento, Italy & [21] \\
\hline Rhinocladiella similis & leaf, twig & Mirandela, Portugal & {$[27,29]$} \\
\hline Rhinocladiella sp. & branch, leaf & Salento, Italy & [21] \\
\hline Saccharata sp. & leaf & Alentejo, Portugal & [32] \\
\hline Sarocladium sp. & branch, leaf & Salento, Italy & [21] \\
\hline \multirow{2}{*}{$\begin{array}{l}\text { Scoliciosporum sp. } \\
\text { Scutellinia sp. }\end{array}$} & branch, leaf & Salento, Italy & [21] \\
\hline & root & Córdoba, Spain & [22] \\
\hline \multirow{2}{*}{$\begin{array}{l}\text { Seimatosporium sp. } \\
\text { Seiridium sp. }\end{array}$} & leaf, twig & Mirandela, Portugal & [29] \\
\hline & twig & Sicily, Italy & [23] \\
\hline \multirow{2}{*}{ Septoria sp. } & leaf, twig & Sicily, Italy & [23] \\
\hline & leaf, twig & Mirandela, Portugal & {$[27,29]$} \\
\hline \multirow{2}{*}{ Sordaria macrospora } & stem, xylem & Majorca, Spain & [30] \\
\hline & twig & Mirandela, Portugal & [27] \\
\hline \multirow{3}{*}{$\begin{array}{l}\text { Sordarla sp. } \\
\text { Sporormiella intermedia } \\
\text { Stagonosporopsis } \\
\text { cucurbitacearum }\end{array}$} & leaf, twig & Mirandela, Portugal & {$[27,29]$} \\
\hline & stem, xylem & Majorca, Spain & [30] \\
\hline & fruit & Karaburun, Turkey & (GenBank) \\
\hline Stemphylium solani & leaf & Alentejo, Portugal & [26] \\
\hline \multirow{2}{*}{ Stemphylium sp. } & leaf, twig & Sicily, Italy & [23] \\
\hline & branch, leaf & Salento, Italy & [21] \\
\hline
\end{tabular}


Table 1. Cont.

\begin{tabular}{|c|c|c|c|}
\hline Endophyte $^{1}$ & Plant Part & Country & Reference \\
\hline \multirow{2}{*}{ Stemphylium vesicarium } & leaf & Alentejo, Portugal & [26] \\
\hline & fruit & Karaburun, Turkey & [GenBank] \\
\hline Stigmatodiscus enigmaticus & xylem & Apulia, Italy & {$[35]$} \\
\hline Talaromyces purpureogenus & root & Portugal & [GenBank] \\
\hline Taphrina sp. & branch, leaf & Salento, Italy & [21] \\
\hline Teratosphaeria sp. & branch, leaf & Salento, Italy & {$[21]$} \\
\hline Tricharina sp. & leaf, twig & Mirandela, Portugal & {$[27,29]$} \\
\hline Tricharina striispora & twig & Mirandela, Portugal & [27] \\
\hline Trichoderma gamsii & root, twig & Bragança district, Portugal & [24] \\
\hline Trichoderma koningii & fruit & Mirandela, Portugal & [28] \\
\hline Trichoderma lixii & root & Bragança district, Portugal & {$[24,40]$} \\
\hline Trichoderma polysporum & stem & Majorca, Spain & {$[30]$} \\
\hline \multirow{3}{*}{ Trichoderma sp. } & root, twig & Bragança district, Portugal & [24] \\
\hline & fruit & Mirandela, Portugal & [28] \\
\hline & leaf, twig & Mirandela, Portugal & [29] \\
\hline Tumularia sp. & leaf, twig & Mirandela, Portugal & {$[27,29]$} \\
\hline Valsa sp. & leaf, twig & Mirandela, Portugal & [29] \\
\hline Valsaria sp. & leaf, twig & Mirandela, Portugal & [29] \\
\hline Venturia sp. & flower buds & Bragança district, Portugal & [34] \\
\hline Verticillium sp. & leaf, twig & Mirandela, Portugal & [29] \\
\hline Wickerhamomyces sp. & branch, leaf & Salento, Italy & [21] \\
\hline Xanthoparmelia sp. & leaf & Salento, Italy & [21] \\
\hline Xanthoria sp. & branch, leaf & Salento, Italy & [21] \\
\hline Xenosonderhenia sp. & branch, leaf & Salento, Italy & [21] \\
\hline \multirow{2}{*}{ Xylaria sp. } & leaf & Piracicaba, Brazil & [33] \\
\hline & twig & Mirandela, Portugal & [27] \\
\hline Zygoascus sp. & branch & Salento, Italy & [21] \\
\hline
\end{tabular}

${ }^{1}$ Species are reported according to the latest accepted name, which might not be the same as the one used in the corresponding reference.

Table 2. Other endophytic fungi reported from Olea europaea.

\begin{tabular}{cccc}
\hline Endophyte $^{\mathbf{1}}$ & Plant Part & Country & Reference \\
\hline Basidiomycota & & & \\
Bullera sp. & leaf & Alentejo, Portugal & {$[32]$} \\
Chondrostereum purpureum & fruit & Mirandela, Portugal & {$[28]$} \\
Colacogloea sp. & branch & Salento, Italy & {$[21]$} \\
Conocybe sp. & root & Córdoba, Spain & {$[22]$} \\
Coprinellus sp. & leaf, twig & Mirandela, Portugal & {$[27,29]$} \\
Coriolopsis sp. & fruit & Bragança district, Portugal & {$[34]$} \\
& leaf, twig & Mirandela, Portugal & {$[29]$} \\
Cryptococcus sp. & leaf & Alentejo, Portugal & {$[32]$} \\
& branch, leaf & Salento, Italy & {$[21]$} \\
Cystofilobasidium sp. & branch & Salento, Italy & {$[21]$} \\
Dioszegia sp. & branch & Salento, Italy & {$[21]$} \\
Entoloma sp. & root & Córdoba, Spain & {$[22]$} \\
Erythrobasidium sp. & leaf & Alentejo, Portugal & {$[32]$} \\
Filobasidium sp. & branch, leaf & Salento, Italy & {$[21]$} \\
Kockovaella sp. & branch & Salento, Italy & {$[21]$} \\
Kondoa sp. & branch, leaf & Salento, Italy & {$[21]$} \\
Lepiota sp. & branch & Salento, Italy & {$[21]$} \\
Malassezia sp. & root & Córdoba, Spain & {$[22]$} \\
Meira sp. & root & Córdoba, Spain & {$[22]$} \\
Moniliophthora sp. & branch, leaf & Salento, Italy & {$[21]$} \\
& branch & Salento, Italy & {$[21]$} \\
& root & Córdoba, Spain & {$[22]$} \\
\hline
\end{tabular}


Table 2. Cont.

\begin{tabular}{|c|c|c|c|}
\hline Endophyte $^{1}$ & Plant Part & Country & Reference \\
\hline Peniophora cinerea & leaf & Alentejo, Portugal & [26] \\
\hline Peniophora lycii & leaf & Alentejo, Portugal & [26] \\
\hline \multirow{2}{*}{ Peniophora sp. } & leaf, twig & Mirandela, Portugal & [29] \\
\hline & branch & Salento, Italy & [21] \\
\hline Phlebiopsis gigantea & leaf & Alentejo, Portugal & [26] \\
\hline Porostereum sp. & leaf, twig & Mirandela, Portugal & [29] \\
\hline Pseudomicrostroma sp. & branch & Salento, Italy & [21] \\
\hline Quambalaria sp. & branch, leaf & Salento, Italy & [21] \\
\hline \multirow{2}{*}{ Rhizoctonia sp. } & stem & Majorca, Spain & [30] \\
\hline & leaf, twig & Sicily, Italy & [23] \\
\hline Rhodotorula mucilaginosa & leaf & Alentejo, Portugal & [26] \\
\hline Sistotrema brinkmannii & shoot & Ljubljana, Slovenia & [37] \\
\hline \multirow{2}{*}{ Sporobolomyces sp. } & leaf & Alentejo, Portugal & [32] \\
\hline & branch, leaf & Salento, Italy & [21] \\
\hline Sporotrichum sp. & leaf & Sicily, Italy & [23] \\
\hline Symmetrospora sp. & branch, leaf & Salento, Italy & [21] \\
\hline \multirow{2}{*}{ Trametes sp. } & leaf, twig & Mirandela, Portugal & {$[27,29]$} \\
\hline & branch, leaf & Salento, Italy & [21] \\
\hline Tremella sp. & branch & Salento, Italy & [21] \\
\hline Tricholoma sp. & leaf, twig & Mirandela, Portugal & [29] \\
\hline Vishniacozyma sp. & branch, leaf & Salento, Italy & [21] \\
\hline Wallemia sp. & leaf & Salento, Italy & [21] \\
\hline Xylobolus annosus & twig & Mirandela, Portugal & [27] \\
\hline \multicolumn{4}{|l|}{ Mucoromycota } \\
\hline Mucor plumbeus & stem, xylem & Majorca, Spain & [30] \\
\hline Mucor racemosus & stem & Majorca, Spain & [30] \\
\hline Rhizopus arrhizus & root & Bragança district, Portugal & [24] \\
\hline Rhizopus sp. & leaf & Alentejo, Portugal & [26] \\
\hline Rhizopus stolonifer & fruit & Karaburun, Turkey & (GenBank) \\
\hline Umbelopsis vinacea & stem & Majorca, Spain & [30] \\
\hline
\end{tabular}

${ }^{1}$ Species are reported according to the latest accepted name, which might not be the same as the one used in the corresponding reference.

With reference to the geographic origin, not surprisingly the great majority of these records come from the Mediterranean region, where olive growing is absolutely dominant in statistical terms with approximately 10.2 million hectares in 2018, corresponding to more than $97 \%$ of the overall surface destined to this crop in the world [44]. In addition to geographic and climatic conditions, differences in the species assortment are related to several factors, such as plant organ (Table 1), phenological stage [34], cultivar [22,27,29,32,35], season and cardinal orientation of samplings [23,24,26,29], isolation procedure, and substrate employed [33].

In timeline terms, the great majority of records have been gathered in the last four years, with a significantly increasing trend. In fact, after just three previous investigations carried out in the island of Majorca (Spain) in 1992 [30], Sicily (Italy) in 2008 [23], and Brazil in 2013 [33], and a couple of extemporary findings from Italy [38,41], the available data have been integrated with 48 new records in 2016, 27 in 2017, 68 in 2018, 107 in 2019, and 73 in the first half of the current year. Several reasons can explain such an escalation. Particularly, the increasingly easier access to the molecular tools has remarkably enhanced the number of isolates which can be taxonomically identified, subverting the old classification procedures which basically relied on the investigators' mycological experience, or on the access to identification services. Previously infrequent or unknown taxa have started being reported thanks to this methodological improvement. However, in many of these records identification was limited at the genus level, particularly when rDNA-ITS sequences only were considered as the genetic marker [21,22,29]. Although acceptable, this reflects a lower significance of the reports, considering that different species within genera such as Alternaria, Aspergillus, Cladosporium, Diaporthe, Fusarium, 
Penicillium, Phoma, etc., may play very different ecological roles. With reference to the identified species, just two common plant associates (Alternaria alternata and Epicoccum nigrum) have more than two records from different locations, indicating that for the time being no species seems to stand out for a regular endophytic association with olive tree.

Another explanation for this incremental trend is the previously introduced emerging awareness of a relationship between endophyte occurrence and pest and pathogen incidences in crops. In the case of olive tree, this concept has been particularly considered with reference to the outbreak of the quick decline syndrome incited by Xylella fastidiosa in southern Italy [45], although no significant associations of any identified endophytes with this bacterium were found in both a high and a low susceptible cultivar in a dedicated study [21]. In another study concerning relationships with another widespread bacterial pathogen, Pseudomonas savastanoi pv. savastanoi causing the olive knot disease, endophytic fungi were found to be more abundant in infected plants. However, this remark particularly involved potential pathogens, such as Alternaria, Cladosporium, Pseudocercospora, Fusarium, and other Nectriaceae [27].

\subsection{Endophytic Fungi as Plant Disease Agents}

The wilt agent Verticillium dahliae is probably the fungal pathogen of olive tree which could be considered to have more strict endophytic implications. Basically, it is considered a hemibiotrophic fungus which colonizes olive trees systemically by spreading through the xylem during a biotrophic phase where it causes no or minimal detrimental effects on plant physiology. Symptoms consisting in chlorotic leaves rolling inward, defoliation, necrosis, and branch desiccation become evident later on [46]. Considering that this more or less enduring latent stage is recognized as a crucial phase of the disease cycle, the recovery of $V$. dahliae from asymptomatic plants is conventionally not referred to a possible merely endophytic status. However, its finding in artificially inoculated plants which became asymptomatic after recovering from infection [47] raises questions on whether its occurrence within olive trees is necessarily related to pathogenicity.

Other known disease agents have been isolated from asymptomatic plants. An inventory of olive tree pathogens compiled in 2014 includes at least 12 species reported in Table 1, namely A. alternata, Alternaria consortialis (=Ulocladium consortiale), Arthrinium phaeospermum, Berkeleyomyces basicola (=Thielaviopsis basicola), Botrytis cinerea, Colletotrichum acutatum, Dothiorella iberica, Epicoccum nigrum, Fusarium oxysporum, Macrophomina phaseolina, Neocosmospora solani (=Fusarium solani) and Neofabraea vagabunda (=N. alba, Phlyctema vagabunda) [48]. In addition to the above-mentioned common associates of olive trees, A. alternata and E. nigrum are also known to develop epiphytically [41], for the other species, most of which are anyway not reported to cause relevant damage to this crop, it is not inferable if their endophytic presence documented in references of Table 1 was eventually preliminary to disease onset.

In addition to the above inventory, more taxa listed in Table 1 were recovered from diseased olive trees, albeit without verifying their involvement in etiology through the Koch's postulates. This is the case of the new species Phaeomoniella oleae, which was originally isolated from black-discolored xylem of wilting branch of a plant infected by X. fastidiosa [49], as well as Conyozyma leucospermi, Nigrospora oryzae and Biscogniauxia mediterranea, which were isolated from twig cankers in California [50]. However, the latter species has been very recently reported as the agent of a charcoal disease in Tunisia [51]. Other recent reports concern Diaporthe ambigua causing twig cankers in Italy [52] and Neofabraea kienholzii causing leaf and shoot lesions in California [53], while Cytospora pruinosa recovered from plants showing branch dieback symptoms in Spain was found not to be pathogenic [54]. Notwithstanding, reports concerning Cytospora [30,35] must be taken with caution, considering recent assessments of pathogenicity on olive tree by a few Cytospora spp., including the novel species C. olivarum $[55,56]$.

The endophytic occurrence of more fungi only identified at the genus level should be better evaluated as well. In addition to Venturia and Verticillium found in Portugal [29,34], this applies to strains in the genera Neofusicoccum and Phaeoacremonium, with reference to records of P. aleophilum and 
several Neofusicoccum spp. as agents of branch dieback and decline of olive trees [50,54,57]. Similar considerations are valid for strains of Diaporthe (=Phomopsis), which are renowned canker agents of common endophytic occurrence on many woody plants [58], Diplodia, Pestalotiopsis, and Phoma, including species which can be disease agents of olive tree [48]. Above all, this concept concerns endophytic strains of Colletotrichum which are frequently reported in asymptomatic plants of both olive tree [27-29,33,59] and other crops, such as citrus [60]. Several Colletotrichum spp. are involved in etiology of olive anthracnose with various degrees of virulence and latency [61-63], including the new species $C$. clavatus to which the findings of $C$. acutatum as endophyte in olive drupes in Italy are likely to be referable [64]. It goes without saying that a more thorough assessment of their endophytic occurrence in the different cropping contexts is expected to have a remarkable impact on the management of anthracnose.

Unlike the above pathogens, the agent of the Dalmatian disease of olives, Botryosphaeria dothidea [65], is missing in the list of endophytic fungi, despite this species is best known as an endophyte of a high number of plants [66]. The fact that its documented occurrence is restricted to diseased drupes is possibly linked to the role as a vector by the cecidomyid Lasioptera berlesiana, a parasitoid of the olive fruit fly Bactrocera oleae [67], considering that $B$. dothidea is constantly associated with many cecidomyid midges [68].

\subsection{Endophytic Fungi as Mutualists}

Many species found as endophytes of olive tree are known to behave as mutualists in crops, based on their ability to contrast pests and pathogens, and/or to promote plant growth; a brief overview of such properties with reference to species included in Table 1 is proposed in this paragraph.

Strains of Trichoderma, mainly reported from Portugal and ascribed to at least four species, have already been experimentally evaluated on olive tree, with reference to both kinds of beneficial effects, particularly for the biocontrol of $V$. dahliae and $N$. solani [18,69-71]. The typical soil fungus Penicillium restrictum has been reported as an antagonist and mycoparasite of several plant pathogens [72], but other Penicillium spp. mentioned in Table 1 are also known as endophytic associates exhibiting antifungal effects [73]. Traditionally known as a biocontrol agent of the grey mold agent B. cinerea [74], Clonostachys rosea (=Bionectria ochroleuca) has also disclosed potential against insects and nematodes [75], likewise Purpureocillium lilacinum [76]. Already employed in the formulation of biopesticides, the latter species has been reported for antagonism against $V$. dahliae on eggplant, along with plant growth promoting effects [77]. Again, found in association with $V$. dahliae in southern Italy [41], Paecilomyces variotii is known for its antagonistic behavior against plant pathogenic fungi in vitro and in vivo [78], as well as nematodes [79]. Species of Cosmospora have been reported as mycoparasites [80], and pathogens of armored scales (Hemiptera, Diaspididae) [81]. Surprisingly, some species of common endophytic occurrence which are basically known as entomopathogens or display a dual biocontrol aptitude against both arthropods and fungi, such as Beauveria bassiana, Metarhizium anisopliae, and Lecanicillium/Akanthomyces spp. [82-84], have not been found as endophytes of olive tree so far.

Based on previous citations as fungal antagonists and producers of bioactive secondary metabolites, other species included in Table 1 may have a role in defensive mutualism [12,15,16,19], such as Chaetomium globosum [85], and species of Paraconiothyrium [86], Alternaria [87], and Epicoccum [88]. Strains of the two latter genera were found at a significantly higher rate in asymptomatic leaves in a survey carried out in Portugal considering the key leaf pathogens of olive tree Venturia oleaginea and Pseudocercospora cladosporioides, which may be indicative of an antagonistic role against the above disease agents [32]. Similar considerations have been advanced for Chromelosporium carneum and other taxa in the Pezizales (e.g., Heydenia and Pyronema); in fact, these fungi were more frequent in plants which did not show symptoms of the olive knot disease [27]. Indeed, their role in suppressing this bacterial disease deserves to be further investigated. 
Finally, other endophytic associates of olive tree are more reputed for their plant growth promoting potential depending on improvement of nutrient availability and/or production of plant hormones, such as auxins by Discosia sp. [89], and gibberellins by Phoma herbarum [90]. Moreover, several yeast species are known for these effects, such as Aureobasidium pullulans, which stands out for its widespread occurrence on the olive phylloplane too [41,91].

\subsection{Endophytic Fungi as Neutral Associates}

The ecological role of the many identified endophytic fungi which were not mentioned in the previous sections requires further assessments. In the absence of any circumstantial evidence, such associations are usually defined as neutral. However, it is hard to accept that this approximate inference is valid for such a high number of taxa; rather, it is likely that at least some of them are going to disclose better defined ecological relationships in future. As an example, the ability by a plant to support endophytic development and reproduction of fungi which are pathogenic towards other plant species has been envisaged to possibly represent an ecological adaptation supporting the competitive attitude of the plant host [92]. In this respect the available data indicate that olive can be the host of species such as Cadophora luteo-olivacea, Curvularia trifolii, Dactylonectria pauciseptata, Didymella macrostoma, Drechslera avenae, Eutypa tetragona, Paraphoma chrysanthemicola, Parastagonospora avenae, Pestalotiopsis guepinii, Pleospora herbarum, Stagonosporopsis cucurbitacearum, and Chondrostereum purpureum, known as pathogens of various crops.

For other fungi already known for their endophytic attitude on several plant species, such as Anthostomella leucospermi, Arcopilus aureus, Chalastospora gossypii, Daldinia concentrica, Endoconidioma populi, Fimetariella rabenhorstii, Nemania aenea, Paraphaeosphaeria sporulosa, Preussia africana, and Sporormiella intermedia, the occurrence on olive tree may be rather interpreted as reflecting a general ecological adaptation to horizontal spread within the phytocoenoses [93].

Because of their recent taxonomic description, other species listed in Table 1 have no significant references in the literature considering their ecological role yet. This is the case of the Dothideomycetes Leptosphaerulina saccharicola [94], Dendrothyrium variisporum [95], Libertasomyces platani [96], and Stigmatodiscus enigmaticus, with the latter representing the founder of the new order Stigmatodiscales [97].

Although lichens are quite common epiphytes of olive trees, the endophytic occurrence of some lichenicolous species is to be remarked with reference to their exclusive finding in the mentioned study concerning the X. fastidiosa epidemic in Salento, Italy [21]. More in detail it is about the genera Absconditella, Biatora, Lecania, Lecanora, Lecidella, Leimonis, Xanthoria, Catillaria, Hyperphyscia, Pyrrhospora, Scoliciosporum and Xanthoparmelia (=Karoowia), with the last five only found in the cultivar Leccino which is known to be resistant to this bacterial disease.

Finally, a mention is deserved for some taxa which have been described as opportunistic human pathogens, such as Cladophialophora, Exophiala, Fusarium musae, Hormonema, Lecythophora, Rhinocladiella similis, and the yeasts Candida, Pichia, Wickerhamomyces, Cryptococcus, Malassezia and Rhodotorula mucilaginosa [98].

\section{Biochemical Properties and Possible Biotechnological Applications}

It is generally accepted that endophytes exert defensive mutualism through biochemical interactions with both the host plant and its pests and pathogens. The ability to release bioactive secondary metabolites and enzymes may ensure direct or indirect antagonistic effects and promote the host's defense reaction. Based on this paradigm, a huge amount of studies concerning microbial endophytes deal with the characterization of products and enzyme complexes and examine perspectives for their biotechnological exploitation $[99,100]$.

So far, the available information concerning endophytic fungi of olive tree is quite limited, and basically concerns known compounds. The acidic terpenoid arundifungin was found as a product of an unidentified Coelomycetes strain recovered as an olive endophyte in Spain, possessing 
antifungal properties which derive from inhibition of glucan synthesis, likewise the better known echinocandin [101]. A strain of Penicillium chrysogenum was found to produce the bioactive indole alkaloids meleagrin, roquefortine $C$, and dehydrohistidyltryptophenyl-diketopiperazine [42]. Moreover, from the same location of the Egyptian oasis of Siwa, an isolate of Penicillium citrinum was reported to produce the polyketide mycotoxin citrinin, along with the pyrrolidine alkaloids 2-(hept-5-enyl)-3-methyl-4-oxo-6,7,8,8a-tetrahydro-4H-pyrrolo[2,1-b]-1,3-oxazine, scalusamide A, and perinadine A [36].

Endophytic fungi recovered from olive leaves and identified as Alternaria sp., Chaetomium sp., Diaporthe sp., Fusarium sp., E. nigrum, and Nigrospora oryzae were found to possess antagonistic properties against $C$. acutatum in vitro, with the latter species displaying the most consistent effects. Such effects were at least in part related to the production of volatile organic compounds (VOCs), such as phenylethyl alcohol, pyrazines, amine, and propanoic acid derivatives [31].

In other cases, investigations have been limited to a preliminary stage considering culture filtrates or extracts from the same. This is the case of culture filtrates obtained from endophytic strains of E. nigrum and R. similis displaying inhibitory effects against $P$. savastanoi pv. savastanoi $[102,103]$. Antibiotic activity against both Gram + and Gram- bacteria has been reported in vitro for strains of Penicillium canescens, Penicillium commune, and A. alternata, with the latter also active against yeasts (Candida spp.) [25]. In the case of A. alternata, the antibiotic effect was also induced by the ethyl acetate extracts from both mycelium and the culture broth, while in the case of P. commune, bioactivity increased after the addition of an olive leaf extract to the medium.

Penicillium commune was again reported for in vitro antagonistic effects against the agent of anthracnose (C. acutatum); such effects were increased by the placement of an olive leaf in the test plates, indicating that interactions might be more dramatic in the contact with plant host [43]. The same research group pointed out inhibitory effects on mycelial growth of this pathogen by endophytic strains of Chondrostereum purpureum, with evident alterations in hyphal structure [28], and of both C. acutatum and V. dahliae by Trichoderma lixii and P. lilacinum [40]. Moreover, endophytic strains of A. pullulans applied at the blooming stage or just prior to harvest were respectively able to reduce incidence of the anthracnose agent during its latency and in post-harvest on the shelf [59]. The latter effect is confirmatory of reports referring to other fruit crops that VOCs released by endophytic strains of this yeast effectively inhibit spore germination of several post-harvest pathogens [104,105].

An olive endophytic strain identified as Daldinia cf. concentrica also showed inhibitory effects against a panel of plant pathogenic fungi and oomycota, which were basically dependent on the production of antimicrobial VOCs. Exposure of dried fruits and grains to these volatiles resulted in their full disinfection preventing the development of molds and suppressed infection by Aspergillus niger in peanuts [39]. In greenhouse experiments VOCs produced by this strain showed bionematicidal activity against the second-stage juveniles of Meloidogyne javanica (67\% reduction in viability). Among these volatiles, 4-heptanone elicited the most consistent effect with $90 \%$ reduction in viability, while egg hatching decreased by $87 \%$. Moreover, the application of a volatile mixture to soil inoculated with $M$. javanica eggs or juveniles significantly reduced galling index in susceptible tomato plants with no effect on root weight [106].

Finally, an interesting ground of investigation has been envisaged for endophytic fungi of olive tree in view of applicative perspectives in the management of X. fastidiosa epidemic spread, based on evidence of either repellent or attractant effects that some species possibly exert against its vector, the meadow spittlebug (Philaenus spumarius) [107].

\section{Conclusions}

Research on endophytic fungi is gradually evolving from a basically descriptive stage to the analysis of factors determining the structure of microbiomes, in the perspective that their manipulation may enable to increase plant protection and productivity. In this respect, it has been observed that a 
better comprehension of the genetic interactions with the host tree and other associated microbes is crucial for the success of practical applications of endophytic fungi in sustainable agriculture [108].

In the case of O. europaea, the increasing number of reports concerning endophytic fungi in the past few years confirm that their spatial-temporal distribution in olive trees has been poorly investigated. However, as for other crops, the accumulating data support the evidence of the substantial impact of this microbial component of biodiversity on fitness of olive tree. As a further example, the recalcitrance to sterilization protocols for in vitro propagation reported in a Slovenian study is indicative that at least some of these fungi are intimately associated to olive plants and adapted for a long-term survival and proliferation in their tissues [37].

Increasing knowledge about functions and dynamics of endophytic communities is fundamental in the aim to exploit use as biocontrol agents. The epidemiological relevance of these microorganisms is basically related to a modulatory role in the spread of cryptogamic diseases. Even when there is no apparent direct interaction with disease agents, the possible effect by endophytic fungi in stimulating plant defense reaction, or more in general to act as plant disease modifiers [109], should not be disregarded. In this respect, data concerning occasional isolations might as well disclose some relevance. Indeed, the role of microbial inoculants on protection, growth stimulation, and productivity is now recognized for olive tree too [14], and the expectancy is high that the applicative use of endophytic fungi may soon become an additional tool in the sustainable management of olive growing.

Author Contributions: Conceptualization, R.N.; resources, R.N. and C.C.; data curation, R.N. and C.C.; writing-original draft preparation, R.N., C.D.V., and C.C.; writing-review and editing, R.N., C.D.V., and C.C.; and funding acquisition, C.C. All authors have read and agreed to the published version of the manuscript.

Funding: This research received no external funding.

Acknowledgments: The contribution of Veronica Vizzarri for some discussion hints is acknowledged.

Conflicts of Interest: The authors declare no conflict of interest.

\section{References}

1. Hirakue, A.; Sugiyama, S. Relationship between foliar endophytes and apple cultivar disease resistance in an organic orchard. Biol. Control 2018, 127, 139-144. [CrossRef]

2. Zornoza, R.; Mataix-Solera, J.; Guerrero, C.; Arcenegui, V.; Mataix-Beneyto, J. Comparison of soil physical, chemical, and biochemical properties among native forest, maintained and abandoned almond orchards in mountainous areas of Eastern Spain. Arid Land Res. Manag. 2009, 23, 267-282. [CrossRef]

3. Palese, A.M.; Magno, R.; Casacchia, T.; Curci, M.; Baronti, S.; Miglietta, F.; Crecchio, C.; Xiloyannis, C.; Sofo, A. Chemical, biochemical and microbiological properties of soils from abandoned and extensively cultivated olive orchards. Sci. World J. 2013, 496278. [CrossRef] [PubMed]

4. Kushwaha, C.P.; Singh, K.P. Crop productivity and soil fertility in a tropical dryland agro-ecosystem: Impact of residue and tillage management. Exp. Agric. 2005, 41, 39-50. [CrossRef]

5. Govaerts, B.; Mezzalama, M.; Sayre, K.D.; Crossa, J.; Lichter, K.; Troch, V.; Vanherck, K.; De Corte, P.; Deckers, J. Long-term consequences of tillage, residue management, and crop rotation on selected soil micro-flora groups in the subtropical highlands. Appl. Soil Ecol. 2008, 38, 197-210. [CrossRef]

6. Di Vaio, C.; Marallo, N.; Marino, G.; Caruso, T. Effect of water stress on dry matter accumulation and partitioning in pot-grown olive trees (cv Leccino and Racioppella). Sci. Hortic. 2013, 164, 172-177. [CrossRef]

7. Cirillo, C.; Russo, R.; Famiani, F.; Di Vaio, C. Investigation on rooting ability of twenty olive cultivars from Southern Italy. Adv. Hortic. Sci. 2017, 31, 311-317.

8. Benitez, E.; Nogales, R.; Campos, M.; Ruano, F. Biochemical variability of olive-orchard soils under different management systems. Appl. Soil Ecol. 2006, 32, 221-231. [CrossRef]

9. Sofo, A.; Ciarfaglia, A.; Scopa, A.; Camele, I.; Curci, M.; Crecchio, C.; Xiloyannis, C.; Palese, A.M. Soil microbial diversity and activity in a Mediterranean olive orchard managed by a set of sustainable agricultural practices. Soil Use Manag. 2014, 30, 160-167. [CrossRef] 
10. Ruano-Rosa, D.; Valverde-Corredor, A.; Gómez-Lama Cabanás, C.; Sesmero, R.; Mercado-Blanco, J. What lies beneath: Root-associated bacteria to improve the growth and health of olive trees. In Soil Biological Communities and Ecosystem Resilience; Lukac, M., Grenni, P., Gamboni, M., Eds.; Springer: Cham, Switzerland, 2017; pp. 107-122.

11. IPCC. 2014: Climate Change 2014: Synthesis Report. Contribution of Working Groups I. II and III to the Fifth Assessment Report of the Intergovernmental Panel on Climate Change; IPCC: Geneva, Switzerland, 2014.

12. Sofo, A.; Manfreda, S.; Dichio, B.; Fiorentino, M.; Xiloyannis, C. The olive tree: A paradigm for drought tolerance in Mediterranean climates. Hydrol. Earth Syst. Sci. 2008, 12, 293-301. [CrossRef]

13. Choudhary, D.K. Microbial rescue to plant under habitat-imposed abiotic and biotic stresses. Appl. Microbiol. Biotechnol. 2012, 96, 1137-1155. [CrossRef] [PubMed]

14. Bizos, G.; Papatheodorou, E.M.; Chatzistathis, T.; Ntalli, N.; Aschonitis, V.G.; Monokrousos, N. The role of microbial inoculants on plant protection, growth stimulation, and crop productivity of the olive tree (Olea europea L.). Plants 2020, 9, 743. [CrossRef] [PubMed]

15. Lata, R.; Chowdhury, S.; Gond, S.; White, J.F. Induction of abiotic stress tolerance in plants by endophytic microbes. Appl. Microbiol. 2018, 66, 268-276. [CrossRef] [PubMed]

16. Yan, L.; Zhu, J.; Zhao, X.; Shi, J.; Jiang, C.; Shao, D. Beneficial effects of endophytic fungi colonization on plants. Appl. Microbiol. Biotechnol. 2019, 103, 3327-3340. [CrossRef]

17. Kasotia, A.; Choundhary, D.K. Role of endophytic microbes in mitigation of abiotic stress in plants. In Emerging Technologies and Management of Crop Stress Tolerance; Ahmad, P., Ed.; Elsevier: Amsterdam, The Netherlands, 2014; Volume 2, pp. 97-108.

18. Dini, I.; Graziani, G.; Gaspari, A.; Fedele, F.L.; Sicari, A.; Vinale, F.; Cavallo, P.; Lorito, M.; Ritieni, A. New strategies in the cultivation of olive trees and repercussions on the nutritional value of the extra virgin olive oil. Molecules 2020, 25, 2345. [CrossRef]

19. Fa, A.N. Endophytic fungi for sustainable agriculture. Microb. Biosyst. 2019, 4, 31-44.

20. Stielow, J.B.; Levesque, C.A.; Seifert, K.A.; Meyer, W.; Iriny, L.; Smits, D.; Renfurm, R.; Verkley, G.J.M.; Groenewald, M.; Chaduli, D.; et al. One fungus, which genes? Development and assessment of universal primers for potential secondary fungal DNA barcodes. Persoonia 2015, 35, 242-263. [CrossRef]

21. Vergine, M.; Meyer, J.B.; Cardinale, M.; Sabella, E.; Hartmann, M.; Cherubini, P.; De Bellis, L.; Luvisi, A. The Xylella fastidiosa-resistant olive cultivar "Leccino" has stable endophytic microbiota during the olive quick decline syndrome (OQDS). Pathogens 2020, 9, 35. [CrossRef]

22. Fernández-González, A.J.; Villadas, P.J.; Cabanás, C.G.L.; Valverde-Corredor, A.; Belaj, A.; Mercado-Blanco, J.; Fernández-López, M. Defining the root endosphere and rhizosphere microbiomes from the World Olive Germplasm Collection. Sci. Rep. 2019, 9, 20423. [CrossRef]

23. Ferraro, V.; Conigliaro, G.; Torta, L.; Burruano, S.; Moschetti, G. Preliminary investigation on the endophytic communities in Olea europaea in Sicily. In Proceedings of the 7th International Conference Integrated Fruit Production, Avignon, France, 27-30 October 2008; pp. 459-463.

24. Martins, F.; Pereira, J.A.; Bota, P.; Bento, A.; Baptista, P. Fungal endophyte communities in above-and belowground olive tree organs and the effect of season and geographic location on their structures. Fungal Ecol. 2016, 20, 193-201. [CrossRef]

25. Malhadas, C.; Malheiro, R.; Pereira, J.A.; de Pinho, P.G.; Baptista, P. Antimicrobial activity of endophytic fungi from olive tree leaves. World J. Microbiol. Biotechnol. 2017, 33, 46. [CrossRef] [PubMed]

26. Materatski, P.; Varanda, C.; Carvalho, T.; Bento Dias, A.; Campos, M.D.; Rei, F.; Félix, M.R. Spatial and temporal variation of fungal endophytic richness and diversity associated to the phyllosphere of olive cultivars. Fungal Biol. 2019, 123, 66-76. [CrossRef] [PubMed]

27. Gomes, T.; Pereira, J.A.; Lino-Neto, T.; Bennett, A.E.; Baptista, P. Bacterial disease induced changes in fungal communities of olive tree twigs depend on host genotype. Sci. Rep. 2019, 9, 5882. [CrossRef] [PubMed]

28. Preto, G.; Martins, F.; Pereira, J.A.; Baptista, P. Fungal community in olive fruits of cultivars with different susceptibilities to anthracnose and selection of isolates to be used as biocontrol agents. Biol. Control 2017, 110, 1-9. [CrossRef]

29. Gomes, T.; Pereira, J.A.; Benhadi, J.; Lino-Neto, T.; Baptista, P. Endophytic and epiphytic phyllosphere fungal communities are shaped by different environmental factors in a Mediterranean ecosystem. Microb. Ecol. 2018, 36, 668-679. [CrossRef] 
30. Fisher, P.J.; Petrini, O.; Petrini, L.E.; Descals, E. A preliminary study of fungi inhabiting xylem and whole stems of Olea europaea. Sydowia 1992, 44, 117-121.

31. Landum, M.C.; Félix, M.R.; Alho, J.; Garcia, R.; Cabrita, M.J.; Rei, F.; Varanda, C.M. Antagonistic activity of fungi of Olea europaea L. against Colletotrichum acutatum. Microbiol. Res. 2016, 183, 100-108. [CrossRef]

32. Varanda, C.M.; Materatski, P.; Landum, M.; Campos, M.D.; Félix, M.D.R. Fungal communities associated with peacock and cercospora leaf spots in olive. Plants 2019, 8, 169. [CrossRef]

33. de Freitas Sia, E.; Marcon, J.; Mazzer Luvizotto, D.; Quecine, M.C.; Tsui, S.; Pereira, J.O.; Pizzirani-Kleiner, A.A.; Azevedo, J.L. Endophytic fungi from the Amazonian plant Paullinia cupana and from Olea europaea isolated using cassava as an alternative starch media source. SpringerPlus 2013, 2, 579.

34. Martins, F.; Pereira, J.A.; Baptista, P. Dynamics of fungal endophytes over different phenological stages of the olive tree host. In Proceedings of the 15th Congress of the Mediterranean Phytopathological Union, Cordoba, Spain, 20-23 June 2017.

35. Hanani, A.; Valentini, F.; Sanzani, S.M.; Gallo, M.; Davino, S.-W.; D’Onghia, A.M. Assessment of the endophytic fungal community in Apulian olive varieties with different potential susceptibilities to Xylella fastidiosa. J. Plant Pathol. 2019, 101, 811.

36. Mady, M.S.; Houssen, W.; Abdou, R.; Haggag, E.G.; El Sayed, K.A. Breast cancer migration and proliferation inhibitory and antibiotic secondary metabolites from the Egyptian olive tree endophytic fungus Penicillium citrinum. J. Advan. Pharm. Res. 2017, 1, 160-170. [CrossRef]

37. Oražem, P.; Celar, F.A.; Bohanec, B. Occurrence of endophytic fungi causing recalcitrance of olive cultivar 'Istrska belica' during shoot culture establishment. Arch. Biol. Sci. 2016, 68, 177-186. [CrossRef]

38. Agosteo, G.E.; Macrì, C.; Taccone, P. Susceptibility of olive cv Itrana to anthracnose. J. Plant Pathol. 2005, 87, 287.

39. Liarzi, O.; Bar, E.; Lewinsohn, E.; Ezra, D. Use of the endophytic fungus Daldinia cf. concentrica and its volatiles as bio-control agents. PLoS ONE 2016, 11, e0168242. [CrossRef] [PubMed]

40. Martins, F.; Pereira, J.A.; Bento, A.; Baptista, P. Potentialities of endophytic fungi of olive tree as biological control agents against Colletotrichum acutatum and Verticillium dahliae. In Endophytes for Plant Protection: The State of the Art; Schneider, C., Leifert, C., Feldmann, F., Eds.; Deutsche Phytomedizinische Gesellschaft: Braunschweig, Germany, 2013; p. 190.

41. Nicoletti, R.; Rinaldi, R. Indagine sulla micoflora del filloplano dell'olivo. Riv. Patol. Veg. 1993, 3, 41-47.

42. Mady, M.S.; Mohyeldin, M.M.; Ebrahim, H.Y.; Elsayed, H.E.; Houssen, W.E.; Haggag, E.G.; Soliman, R.F.; El Sayed, K.A. The indole alkaloid meleagrin, from the olive tree endophytic fungus Penicillium chrysogenum, as a novel lead for the control of c-Met-dependent breast cancer proliferation, migration and invasion. Bioorg. Med. Chem. 2016, 24, 113-122. [CrossRef]

43. Martins, F.; Pereira, J.A.; Bento, A.; Baptista, P. Plant-mediated effects on antagonistic activity of endophytic fungi towards olive fungal diseases. In Endophytes for Plant Protection: The State of the Art; Schneider, C., Leifert, C., Feldmann, F., Eds.; Deutsche Phytomedizinische Gesellschaft: Braunschweig, Germany, 2013; pp. 127-128.

44. FAOSTAT Online Database. Available online: http://faostat.fao.org/ (accessed on 1 July 2020).

45. Zicca, S.; De Bellis, P.; Masiello, M.; Saponari, M.; Saldarelli, P.; Boscia, D.; Sisto, A. Antagonistic activity of olive endophytic bacteria and of Bacillus spp. strains against Xylella fastidiosa. Microbiol. Res. 2020, 236, 126467. [CrossRef]

46. López-Escudero, F.J.; Mercado-Blanco, J. Verticillium wilt of olive: A case study to implement an integrated strategy to control a soil-borne pathogen. Plant Soil 2011, 344, 1-50. [CrossRef]

47. Mercado-Blanco, J.; Rodríguez-Jurado, D.; Pérez-Artés, E.; Jiménez-Díaz, R.M. Detection of the nondefoliating pathotype of Verticillium dahliae in infected olive plants by nested PCR. Plant Pathol. 2001, 50, 609-619. [CrossRef]

48. Chliyeh, M.; Touati, J.; Selmaoui, K.; Touhami, A.O.; Filali-Maltouf, A.; El Modafar, C.; Douira, A. Bibliographic inventory of the olive tree (Olea europaea L.) fungal diseases in the world. Int. J. Pure Appl. Biosci. 2014, 2, $46-79$.

49. Crous, P.W.; Groenewald, J.Z.; Nigro, F.; Antelmi, I. Fungal planet description sheets 351—Pseudophaeomoniella Nigro, Antelmi \& Crous, gen. nov. Persoonia 2015, 34, 224-227.

50. Úrbez-Torres, J.R.; Peduto, F.; Vossen, P.M.; Krueger, W.H.; Gubler, W.D. Olive twig and branch dieback: Etiology, incidence, and distribution in California. Plant Dis. 2013, 97, 231-244. [CrossRef] [PubMed] 
51. Gharbi, Y.; Ennouri, K.; Bouazizi, E.; Cheffi, M.; Triki, M.A. First report of charcoal disease caused by Biscogniauxia mediterranea on Olea europaea in Tunisia. J. Plant Pathol. 2020. [CrossRef]

52. Frisullo, S.; Elshafie, H.S.; Mang, S.M. First report of two Phomopsis species on olive trees in Italy. J. Plant Pathol. 2015, 97, 401.

53. Trouillas, F.P.; Nouri, M.T.; Lawrence, D.P.; Moral, J.; Travadon, R.; Aegerter, B.J.; Lightle, D. Identification and characterization of Neofabraea kienholzii and Phlyctema vagabunda causing leaf and shoot lesions of olive in California. Plant Dis. 2019, 103, 3018-3030. [CrossRef]

54. Moral, J.; Agustí-Brisach, C.; Pérez-Rodríguez, M.; Xaviér, C.; Raya, M.C.; Rhouma, A.; Trapero, A. Identification of fungal species associated with branch dieback of olive and resistance of table cultivars to Neofusicoccum mediterraneum and Botryosphaeria dothidea. Plant Dis. 2017, 101, 306-316. [CrossRef]

55. Lawrence, D.P.; Holland, L.A.; Nouri, M.T.; Travadon, R.; Abramians, A.; Michailides, T.J.; Trouillas, F.P. Molecular phylogeny of Cytospora species associated with canker diseases of fruit and nut crops in California, with the descriptions of ten new species and one new combination. IMA Fungus 2018, 9, 333-369. [CrossRef]

56. Úrbez-Torres, J.R.R.; Lawrence, D.P.; Peduto Hand, F.; Trouillas, F. Olive twig and branch dieback in California caused by Cytospora oleicola and the newly described species Cytospora olivarum sp. nov. Plant Dis. 2020, 104. [CrossRef]

57. Carlucci, A.; Raimondo, M.L.; Cibelli, F.; Phillips, A.J.; Lops, F. Pleurostomophora richardsiae, Neofusicoccum parvum and Phaeoacremonium aleophilum associated with a decline of olives in southern Italy. Phytopathol. Medit. 2013, 52, 517-527.

58. Udayanga, D.; Liu, X.; McKenzie, E.H.C.; Chukeatirote, E.; Bahkali, A.H.A.; Hyde, K.D. The genus Phomopsis: Biology, applications, species concepts and names of common phytopathogens. Fungal Divers. 2011, 50, 189-225. [CrossRef]

59. Nigro, F.; Antelmi, I.; Labarile, R.; Sion, V.; Pentimone, I. Biological control of olive anthracnose. Acta Hortic. 2018, 439-444. [CrossRef]

60. Nicoletti, R. Endophytic fungi of citrus plants. Agriculture 2019, 9, 247. [CrossRef]

61. Cacciola, S.O.; Faedda, R.; Sinatra, F.; Agosteo, G.E.; Schena, L.; Frisullo, S.; di San Lio, G.M. Olive anthracnose. J. Plant Pathol. 2012, 94, 29-44.

62. Schena, L.; Mosca, S.; Cacciola, S.O.; Faedda, R.; Sanzani, S.M.; Agosteo, G.E.; Sergeeva, V.; di San Lio, G.M. Species of the Colletotrichum gloeosporioides and C. boninense complexes associated with olive anthracnose. Plant Pathol. 2014, 63, 437-446. [CrossRef]

63. Msairi, S.; Chliyeh, M.; Touhami, A.O.; El Alaoui, A.; Selmaoui, K.; Benkirane, R.; Filali-Maltouf, A.; El Modafar, C.; Douira, A. First report of Colletotrichum lupini causing anthracnose disease on the olive fruits in Morocco. Plant Cell Biotechnol. Mol. Biol. 2020, 21, 1-11.

64. Faedda, R.; Agosteo, G.E.; Schena, L.; Mosca, S.; Frisullo, S.; di San Lio, G.M.; Cacciola, S.O. Colletotrichum clavatum sp. nov. identified as the causal agent of olive anthracnose in Italy. Phytopathol. Medit. 2011, 50, 283-302.

65. Phillips, A.J.L.; Rumbos, I.C.; Alves, A.; Correia, A. Morphology and phylogeny of Botryosphaeria dothidea causing fruit rot of olives. Mycopathologia 2005, 159, 433-439. [CrossRef] [PubMed]

66. Slippers, B.; Wingfield, M.J. Botryosphaeriaceae as endophytes and latent pathogens of woody plants: Diversity, ecology and impact. Fungal Biol. Rev. 2007, 21, 90-106. [CrossRef]

67. Latinović, J.; Hrnčić, S.; Perović, T.; Latinović, N. Botryosphaeria dothidea-causal agent of olive fruit rot-pathogen of wounds or not? IOBC-WPRS Bull. 2014, 108, 35-38.

68. Zimowska, B.; Okoń, S.; Becchimanzi, A.; Krol, E.D.; Nicoletti, R. Phylogenetic characterization of Botryosphaeria strains associated with Asphondylia galls on species of Lamiaceae. Diversity 2020, $12,41$. [CrossRef]

69. Carrero-Carrón, I.; Trapero-Casas, J.L.; Olivares-García, C.; Monte, E.; Hermosa, R.; Jiménez-Díaz, R.M. Trichoderma asperellum is effective for biocontrol of Verticillium wilt in olive caused by the defoliating pathotype of Verticillium dahliae. Crop Prot. 2016, 88, 45-52. [CrossRef]

70. Ruano-Rosa, D.; Prieto, P.; Rincón, A.M.; Gómez-Rodríguez, M.V.; Valderrama, R.; Barroso, J.B.; Mercado-Blanco, J. Fate of Trichoderma harzianum in the olive rhizosphere: Time course of the root colonization process and interaction with the fungal pathogen Verticillium dahliae. BioControl 2016, 61, 269-282. [CrossRef] 
71. Ben Amira, M.; Lopez, D.; Triki Mohamed, A.; Khouaja, A.; Chaar, H.; Fumanal, B.; Gousset-Dupont, A.; Bonhomme, L.; Label, P.; Goupil, P.; et al. Beneficial effect of Trichoderma harzianum strain Ths97 in biocontrolling Fusarium solani causal agent of root rot disease in olive trees. Biol. Control 2017, 110, 70-78. [CrossRef]

72. Nicoletti, R.; De Stefano, M. Penicillium restrictum as an antagonist of plant pathogenic fungi. Dyn. Biochem. Process Biotechnol. Mol. Biol. 2012, 6, 61-69.

73. Nicoletti, R.; Fiorentino, A.; Scognamiglio, M. Endophytism of Penicillium species in woody plants. Open Mycol. J. 2014, 8, 1-26. [CrossRef]

74. Sutton, J.C.; Li, D.W.; Peng, G.; Yu, H.; Zhang, P.G.; Valdebenito-Sanhueza, R.M. Gliocladium roseum-A versatile adversary of Botrytis cinerea in crops. Plant Dis. 1997, 81, 316-328. [CrossRef]

75. Sun, Z.B.; Li, S.D.; Ren, Q.; Xu, J.L.; Lu, X.; Sun, M.H. Biology and applications of Clonostachys rosea. J. Appl. Microbiol. 2020. [CrossRef]

76. Fiedler, Ż.; Sosnowska, D. Nematophagous fungus Paecilomyces lilacinus (Thom) Samson is also a biological agent for control of greenhouse insects and mite pests. BioControl 2007, 52, 547-558. [CrossRef]

77. Lan, X.; Zhang, J.; Zong, Z.; Ma, Q.; Wang, Y. Evaluation of the biocontrol potential of Purpureocillium lilacinum QLP12 against Verticillium dahliae in eggplant. BioMed Res. Int. 2017, 2017, 4101357. [CrossRef]

78. Rodrigo, S.; Santamaria, O.; Halecker, S.; Lledó, S.; Stadler, M. Antagonism between Byssochlamys spectabilis (anamorph Paecilomyces variotii) and plant pathogens: Involvement of the bioactive compounds produced by the endophyte. Ann. Appl. Biol. 2017, 171, 464-476. [CrossRef]

79. Al-Qasim, M.; Abu-Gharbieh, W.; Assas, K. Nematophagal ability of Jordanian isolates of Paecilomyces variotii on the root-knot nematode Meloidogyne javanica. Nematol. Medit. 2009, 37, 53-57.

80. Herrera, C.S.; Hirooka, Y.; Chaverri, P. Pseudocospeciation of the mycoparasite Cosmospora with their fungal hosts. Ecol. Evol. 2016, 6, 1504-1514. [CrossRef] [PubMed]

81. Mauchline, N.; Hallett, I.; Hill, G.; Casonato, S. Process of infection of armored scale insects (Diaspididae) by an entomopathogenic Cosmospora sp. J. Invert. Pathol. 2011, 108, 46-51. [CrossRef] [PubMed]

82. McKinnon, A.C.; Saari, S.; Moran-Diez, M.E.; Meyling, N.V.; Raad, M.; Glare, T.R. Beauveria bassiana as an endophyte: A critical review on associated methodology and biocontrol potential. BioControl 2017, 62, 1-17. [CrossRef]

83. Behie, S.W.; Jones, S.J.; Bidochka, M.J. Plant tissue localization of the endophytic insect pathogenic fungi Metarhizium and Beauveria. Fungal Ecol. 2015, 13, 112-119. [CrossRef]

84. Nicoletti, R.; Becchimanzi, A. Endophytism of Lecanicillium and Akanthomyces. Agriculture 2020, 10, 205. [CrossRef]

85. Aswini, C. A review on Chaetomium globosum is versatile weapons for various plant pathogens. J. Pharmocognosy Phytochem. 2019, 8, 946-949.

86. Verkley, G.J.; da Silva, M.; Wicklow, D.T.; Crous, P.W. Paraconiothyrium, a new genus to accommodate the mycoparasite Coniothyrium minitans, anamorphs of Paraphaeosphaeria, and four new species. Stud. Mycol. 2004, 50, 323-335.

87. Lou, J.; Fu, L.; Peng, Y.; Zhou, L. Metabolites from Alternaria fungi and their bioactivities. Molecules 2013, 18, 5891-5935. [CrossRef]

88. Braga, R.M.; Padilla, G.; Araújo, W.L. The biotechnological potential of Epicoccum spp.: Diversity of secondary metabolites. Crit. Rev. Microbiol. 2018, 44, 759-778. [CrossRef]

89. Rahi, P.; Vyas, P.; Sharma, S.; Gulati, A.; Gulati, A. Plant growth promoting potential of the fungus Discosia sp. FIHB 571 from tea rhizosphere tested on chickpea, maize and pea. Indian J. Microbiol. 2009, 49, 128-133. [CrossRef] [PubMed]

90. Hamayun, M.; Khan, S.A.; Khan, A.L.; Rehman, G.; Sohn, E.Y.; Shah, A.A.; Kim, S.K.; Joo, G.J.; Lee, I.J. Phoma herbarum as a new gibberellin-producing and plant growth-promoting fungus. J. Microbiol. Biotechnol. 2009, 19, 1244-1249. [PubMed]

91. Abdelfattah, A.; Li Destri Nicosia, M.G.; Cacciola, S.O.; Droby, S.; Schena, L. Metabarcoding analysis of fungal diversity in the phyllosphere and carposphere of olive (Olea europaea). PLoS ONE 2015, 10, e0131069. [CrossRef]

92. Aschehoug, E.T.; Metlen, K.L.; Callaway, R.M.; Newcombe, G. Fungal endophytes directly increase the competitive effects of an invasive forb. Ecology 2012, 93, 3-8. [CrossRef] 
93. Rodriguez, R.J.; White, J.F., Jr.; Arnold, A.E.; Redman, A.R.A. Fungal endophytes: Diversity and functional roles. New Phytol. 2009, 182, 314-330. [CrossRef]

94. Phookamsak, R.; Liu, J.K.; Chukeatirote, E.; McKenzie, E.H.; Hyde, K.D. Phylogeny and morphology of Leptosphaerulina saccharicola sp. nov. and Pleosphaerulina oryzae and relationships with Pithomyces. Cryptogam. Mycol. 2013, 34, 303-319. [CrossRef]

95. Verkley, G.J.M.; Dukik, K.; Renfurm, R.; Göker, M.; Stielow, J.B. Novel genera and species of coniothyrium-like fungi in Montagnulaceae (Ascomycota). Persoonia 2014, 32, 25-51. [CrossRef] [PubMed]

96. Crous, P.W.; Wingfield, M.J.; Burgess, T.I.; Hardy, G.E.S.J.; Crane, C.; Barrett, S.; Cano-Lira, J.F.; Le Roux, J.J.; Thangavel, R.; Guarro, J.; et al. Fungal planet description sheets: 469-557. Persoonia 2016, 37, $218-403$. [CrossRef]

97. Voglmayr, H.; Gardiennet, A.; Jaklitsch, W.M. Asterodiscus and Stigmatodiscus, two new apothecial dothideomycete genera and the new order Stigmatodiscales. Fungal Divers. 2016, 80, 271-284. [CrossRef]

98. Sherrington, S.L.; Kumwenda, P.; Kousser, C.; Hall, R.A. Host sensing by pathogenic fungi. Advan. Appl. Microbiol. 2018, 102, 159-221.

99. Nicoletti, R.; Fiorentino, A. Plant bioactive metabolites and drugs produced by endophytic fungi of Spermatophyta. Agriculture 2015, 5, 918-970. [CrossRef]

100. Yan, L.; Zhao, H.; Zhao, X.; Xu, X.; Di, Y.; Jiang, C.; Shi, J.; Shao, D.; Huang, Q.; Yang, H.; et al. Production of bioproducts by endophytic fungi: Chemical ecology, biotechnological applications, bottlenecks, and solutions. Appl. Microbiol. Biotechnol. 2018, 102, 6279-6298. [CrossRef] [PubMed]

101. Cabello, A.M.; Platas, G.; Collado, J.; Díez, T.M.; Martín, I.; Vicente, F.; Meinz, M.; Onishi, J.C.; Thompson, C.D.J.; Kurtz, M.B.; et al. Arundifungin, a novel antifungal compound produced by fungi: Biological activity and taxonomy of the producing organisms. Int. Microbiol. 2001, 4, 93-102. [CrossRef] [PubMed]

102. Gomes, T.; Pereira, J.A.; Lino-Neto, T.; Baptista, P. Endophytic and epiphytic fungal community associated to olive tree differ in antagonistic activity against Pseudomonas savastanoi pv. savastanoi. In Proceedings of the 15th Congress Mediterranean Phytopathological Union, Cordoba, Spain, 20-23 June 2017; pp. 202-203.

103. Berardo, C.; Bulai, I.M.; Venturino, E.; Baptista, P.; Gomes, T. Modeling the endophytic fungus Epicoccum nigrum action to fight the "olive knot" disease caused by Pseudomonas savastanoi pv. savastanoi (Psv) bacteria in Olea europaea L. Trees. In Trends in Biomathematics: Modeling, Optimization and Computational Problems; Springer: Cham, Switzerland, 2018; pp. 189-207.

104. Di Francesco, A.; Ugolini, L.; Lazzeri, L.; Mari, M. Production of volatile organic compounds by Aureobasidium pullulans as a potential mechanism of action against postharvest fruit pathogens. Biol. Control 2015, 81, 8-14. [CrossRef]

105. Don, S.Y.; Schmidtke, L.M.; Gambetta, J.M.; Steel, C.C. Aureobasidium pullulans volatilome identified by a novel, quantitative approach employing SPME-GC-MS, suppressed Botrytis cinerea and Alternaria alternata in vitro. Sci. Rep. 2020, 10, 4498.

106. Liarzi, O.; Bucki, P.; Miyara, S.B.; Ezra, D. Bioactive volatiles from an endophytic Daldinia cf. concentrica isolate affect the viability of the plant parasitic nematode Meloidogyne javanica. PLoS ONE 2016, 11, e0168437.

107. Cameirão, C.; Fernandes, G.; Martins, F.; Pereira, J.A.; Baptista, P. The effect of Philaenus spumarius feeding on the endophytic fungi community of Coleostephus myconis and its possible applicability in the insect biocontrol. In Proceedings of the 8th Meeting of the IOBC-WPRS Working Group 'Integrated Protection of Olive Crops', Florence, Italy, 4-7 June 2018.

108. Schlaeppi, K.; Bulgarelli, D. The plant microbiome at work. Mol. Plant Microbe Interact. 2015, 28, $212-217$. [CrossRef]

109. Busby, P.E.; Ridout, M.; Newcombe, G. Fungal endophytes: Modifiers of plant disease. Plant Mol. Biol. 2016, 90, 645-655. [CrossRef]

(C) 2020 by the authors. Licensee MDPI, Basel, Switzerland. This article is an open access article distributed under the terms and conditions of the Creative Commons Attribution (CC BY) license (http://creativecommons.org/licenses/by/4.0/). 